\title{
When and How to Treat Subthreshold Depression
}

Kurt Kroenke, MD

Depressive disorders are present in about $10 \%$ of primary care patients and account for more years lived with disability than any single disease. ${ }^{1,2}$ Nearly three-quarters of all outpatient visits for depression are to primary care clinicians rather than to mental health specialists. ${ }^{3}$

Related article page 728

Collaborative care is a therapeutic intervention in which behavioral health is integrated into primary care, most commonly using a nurse care manager to monitor depressive symptoms in depressed patients and adjust treatment under the supervision of a psychiatrist. Many of the nurse contacts are conducted by telephone, thereby increasing the efficiency of collaborative care. Although collaborative care has been demonstrated to improve depression in more than 80 randomized clinical trials, ${ }^{4,5}$ most trials have targeted major depression.

Major depression requires the presence of at least 5 of 9 criterion symptoms of depression for 2 weeks or longer, with at least 1 of the symptoms being depressed mood or anhedonia. ${ }^{6}$ In comparison, subthreshold depression (also called minor or subsyndromal depression) is the presence of 2 to 4 criterion symptoms of depression for 2 weeks or longer with at least 1 of the core symptoms (depressed mood or anhedonia). ${ }^{6,7}$ Alternative definitions of subthreshold depression use a severity cut point on a depression scale or vary in duration and core symptom requirements but typically require the absence of major depression. Only a small number of therapeutic trials have targeted subthreshold depression, and results have been mixed. ${ }^{6,8}$ Identifying effective therapies for subthreshold depression is important because many patients with subthreshold depression have persistent depressive symptoms at 12-month follow-up, a third to half report moderate functional impairment, and at least $10 \%$ to $20 \%$ progress to major depression..$^{6,7,9,10}$

In this issue of JAMA, the CASPER trial by Gilbody et $\mathrm{al}^{11}$ provides the first evidence that collaborative care may benefit patients with subthreshold depression. In this pragmatic clinical trial conducted in the United Kingdom, the authors randomized 705 adults aged 65 years or older with subthreshold depression to either a collaborative care intervention or usual primary care. The collaborative care treatment consisted of 8 weekly 30-minute sessions of behavioral activation administered by a care manager with a background in mental health nursing or psychology who was supervised by a mental health professional. Behavioral activation is a psychological intervention that encourages increased social interactions and engagement in pleasur- able, rewarding activities. The first session was face-to-face, and subsequent sessions were delivered by telephone.

Several important findings emerged from the CASPER trial. First, improvement on the 9-item Patient Health Questionnaire depression score (PHQ-9, which ranges from 0-27) at 4 months was 1.3 points greater in the collaborative care group compared with the usual care group (the PHQ-9 score was 7.8 in both groups at baseline and declined to 5.4 in the collaborative care group and 6.7 in the usual care group). This difference was sustained at 12-month follow-up and represents an effect size of 0.3 , consistent with a small to moderate clinical improvement and comparable with the average effect size demonstrated in collaborative care trials for major depression. ${ }^{4,5}$ This is notable because the mean baseline PHQ-9 score among patients in this trial was only 7.8 (median, 7), meaning that there was a limit to how much improvement could be achieved given that a score of less than 5 corresponds to minimal depressive symptoms.

A second important finding was that progression to threshold-level depression (defined in this trial as a PHQ-9 score $\geq 10$ ) was not different between the 2 groups at 4 months (17.2\% vs $23.5 \%)$ but was significantly less frequent in the collaborative care group compared with usual care at 12 months (15.7\% vs $27.8 \%$ ). This represents a relative risk of 0.65 (95\% CI, 0.46-0.91). Third, the collaborative care intervention improved anxiety, functional status, and several other secondary outcomes compared with usual care. The effect size for anxiety was 0.23 at 4 -month follow-up, a significant between-group difference that was sustained at 12-month follow-up and comparable with the average effect sizes of 0.30 to 0.33 reported in other studies examining collaborative care for anxiety. ${ }^{4,5}$ The reduction in anxiety is especially important because anxiety has a similar prevalence as depression in primary care practice and is present in a third to half of patients with depression. ${ }^{1}$

Another important aspect of the CASPER intervention was that it was brief and relatively inexpensive. The intervention consisted of 8 telephone sessions lasting $30 \mathrm{~min}-$ utes each delivered by care managers with less psychological training than psychologists or other mental health professionals. Other study strengths included the large sample size; the low rate of antidepressant therapy, which was balanced among groups (thus minimizing the effect of antidepressants as a cointervention and potential confounder); and a sensitivity analysis showing that effectiveness did not vary among care managers. Although attrition was higher in the collaborative care group than in the usual care group, imputed analyses accounting for this difference did not change the results. 
The core treatment in the CASPER trial was behavioral activation, for which a previous meta-analysis of 26 trials involving 1524 depressed patients also showed a reduction in depression. ${ }^{12}$ Strong evidence for the effectiveness of behavioral activation was provided by the recent COBRA trial, in which 440 adults with major depression were randomized to either behavioral activation or cognitive behavioral therapy. ${ }^{13}$ Each group received an average of 12 onehour in-person sessions, and behavioral activation was found to be noninferior to cognitive behavioral therapy for the outcome of depression. A meta-analysis of brief psychological therapies (typically $\leq 8$ sessions) found that cognitive behavioral therapy (13 trials), problem-solving therapy (12 trials), and counseling (8 trials) were all associated with improved depression outcomes in primary care, with effect sizes ranging from 0.21 to $0.33 .{ }^{14}$ Thus, behavioral activation can now be added to the list of brief therapies that might be considered for treating depressed patients in primary care.

A recent German trial also found benefits in treating subthreshold depression: 406 adults with subthreshold depression were randomized to either a web-based guided intervention (cognitive behavioral and problem-solving therapy supported by an online trainer) or a web-based psychoeducation control program. ${ }^{15}$ Fewer patients in the intervention group progressed to major depression at 12-month follow-up (27\% vs $41 \%$ ), resulting in a number needed to treat of 5.9 to avoid 1 new case of major depression (compared with a number needed to treat of 8.3 in CASPER). Costeffectiveness results for the CASPER trial are planned but not yet available. Notably, collaborative care interventions for major depression are cost-effective for the outcome of quality-adjusted life-years. ${ }^{5,16}$ Whether this is also true for collaborative care interventions that target subthreshold depression, for which the absolute amount of improvement is less, needs to be evaluated.

Do results of these recent trials mean that clinicians should expand therapy beyond major depression to include active treatment of subthreshold depression? There are several factors to consider. First, the way in which subthreshold depression is defined varies across studies. ${ }^{7}$ The lack of a consensus on the definition of subthreshold depression can complicate comparisons across treatment trials that define subthreshold depression differently.

Second, the rate at which subthreshold depression progresses to major depression varies. Although the progression rate in the control groups of the CASPER and German trials ranged from $28 \%$ to $41 \%$ over 12 months, ${ }^{11,15}$ other studies suggest a lower progression rate of $10 \%$ to $20 \%$. ${ }^{6,9}$ The higher progression rates in the CASPER and German studies may be because trial participants had a moderate degree of functional impairment. Previous research has shown that the subgroup of patients with subthreshold depression and functional impairment is the most likely to benefit from active treatment. ${ }^{6,17}$ It is likely that if all patients with subthreshold depression were actively treated regardless of level of impairment, the magnitude of benefit would be less and the number needed to treat larger.
Third, patient preferences must be considered. One study of 1025 consecutive patients presenting to 19 general practices in New Zealand found that adding the question to depression screening "Is this something for which you would like help?" markedly decreased the number of false-positive depression diagnoses. ${ }^{18}$ Although this study focused on diagnosis rather than treatment, it is possible that patients with subthreshold depression who do not desire treatment for their symptoms are less likely to benefit.

Fourth, the duration of subthreshold depression and the context in which it occurs should be considered. For example, individuals who experience adjustment reactions to adverse life events often have short-duration depressive symptoms that resolve without active therapy. Thus, there is a role for watchful waiting in subthreshold depression of recent onset. Patients could be asked to monitor their symptoms (possibly by completing a home-based PHQ-9 or other depression scale) and, if they fail to improve within a reasonable period (eg, 1-3 months), to make a follow-up appointment. Alternatively, they could be scheduled for a follow-up telephone call or, with advances in automated monitoring, be prompted to complete a web-based depression scale.

The need to carefully weigh the benefits of treating individuals who have symptoms but fall short of meeting criteria for major depression is not unique to subthreshold depression but is applicable to prediabetes, mild obesity, statins for primary prevention, and numerous other health care decisions in individuals with mild disease. In determining eligibility for the CASPER trial, the ratio of patients with subthreshold to major depression was 4:1. Before expanding treatment beyond major depression to the larger number of patients with subthreshold depression, further research to identify those most likely to benefit is warranted.

Fifth, further study also is needed to determine whether antidepressants have any role as an alternate therapy for subthreshold depression. While antidepressants have frequently not been better than placebo in randomized trials of subthreshold depression, ${ }^{8}$ access to evidence-based psychological therapies varies across health care settings. Individual patient preferences for medication vs nonpharmacological treatments also vary. Limited evidence suggests that antidepressants might be beneficial in patients with more severe subthreshold depression, such as those with functional impairment or suicidal ideation. ${ }^{6,17,19}$ Currently, evidence is stronger for brief psychological therapies when a treatment intervention is considered for subthreshold depression.

In summary, CASPER provides new evidence that collaborative care improves outcomes for at least some patients with subthreshold depression. A principal component of treatment in CASPER was behavioral activation. Other brief psychological therapies such as cognitive behavioral therapy and problem-solving therapy have also proven effective in some studies. Accessibility of these psychological treatments can be enhanced by telephone or web-based delivery. Patients with persistent symptoms, functional impairment, and a desire for treatment may particularly benefit. 


\section{ARTICLE INFORMATION}

Author Affiliations: VA Health Services Research and Development Service Center for Health Communication and Information, Regenstrief Institute, Indianapolis, Indiana; Indiana University School of Medicine, Indianapolis.

Corresponding Author: Kurt Kroenke, MD, Regenstrief Institute, Room 221, 1101 W 10th St, Indianapolis, IN 46202 (kkroenke@regenstrief.org)

Conflict of Interest Disclosures: The author has completed and submitted the ICMJE Form for Disclosure of Potential Conflicts of Interest and none were reported.

\section{REFERENCES}

1. Löwe B, Spitzer RL, Williams JB, Mussell M, Schellberg D, Kroenke K. Depression, anxiety and somatization in primary care: syndrome overlap and functional impairment. Gen Hosp Psychiatry. 2008;30(3):191-199.

2. Murray CJ, Atkinson C, Bhalla K, et al; US Burden of Disease Collaborators. The state of US health, 1990-2010: burden of diseases, injuries, and risk factors. JAMA. 2013;310(6):591-608.

3. Olfson M, Blanco C, Marcus SC. Treatment of adult depression in the United States. JAMA Intern Med. 2016;176(10):1482-1491.

4. Archer J, Bower P, Gilbody S, et al. Collaborative care for depression and anxiety problems. Cochrane Database Syst Rev. 2012;10:CD006525.

5. Reed SJ, Shore KK, Tice JA. Effectiveness and value of integrating behavioral health into primary care. JAMA Intern Med. 2016;176(5):691-692.
6. Kroenke K. Minor depression: midway between major depression and euthymia. Ann Intern Med. 2006;144(7):528-530.

7. Rodríguez MR, Nuevo R, Chatterji S, Ayuso-Mateos JL. Definitions and factors associated with subthreshold depressive conditions: a systematic review. BMC Psychiatry. 2012;12:181.

8. Baumeister $\mathrm{H}$. Inappropriate prescriptions of antidepressant drugs in patients with subthreshold to mild depression: time for the evidence to become practice. J Affect Disord. 2012;139(3):240 243.

9. Lyness JM, Heo M, Datto CJ, et al. Outcomes of minor and subsyndromal depression among elderly patients in primary care settings. Ann Intern Med. 2006;144(7):496-504.

10. Jaffe A, Froom J, Galambos N. Minor depression and functional impairment. Arch Fam Med. 1994:3(12):1081-1086.

11. Gilbody S, Lewis H, Adamson J, et al. Effect of collaborative care vs usual care on depressive symptoms in older adults with subthreshold depression: the CASPER randomized clinical trial. JAMA. doi:10.1001/jama.2017.0130

12. Ekers $D$, Webster $L$, Van Straten $A$, Cuijpers $P$, Richards D, Gilbody S. Behavioural activation for depression: an update of meta-analysis of effectiveness and sub group analysis. PLoS One. 2014;9(6):e100100.

13. Richards DA, Ekers D, McMillan D, et al. Cost and outcome of behavioural activation vs cognitive behavioural therapy for depression (COBRA): a randomised, controlled, non-inferiority trial. Lancet. 2016;388(10047):871-880.

14. Cape J, Whittington $C$, Buszewicz M, Wallace $P$, Underwood L. Brief psychological therapies for anxiety and depression in primary care: meta-analysis and meta-regression. BMC Med. 2010;8:38.

15. Buntrock C, Ebert DD, Lehr D, et al. Effect of a web-based guided self-help intervention for prevention of major depression in adults with subthreshold depression: a randomized clinical trial. JAMA. 2016;315(17):1854-1863.

16. Jacob V, Chattopadhyay SK, Sipe TA, Thota AB Byard GJ, Chapman DP; Community Preventive Services Task Force. Economics of collaborative care for management of depressive disorders: a community guide systematic review. Am J Prev Med. 2012;42(5):539-549.

17. Ackermann RT, Williams JW Jr. Rational treatment choices for non-major depressions in primary care: an evidence-based review. J Gen Intern Med. 2002;17(4):293-301.

18. Arroll B, Goodyear-Smith F, Kerse N, Fishman T, Gunn J. Effect of the addition of a "help" question to two screening questions on specificity for diagnosis of depression in general practice: diagnostic validity study. BMJ. 2005;331(7521):884.

19. Bruce ML, Ten Have TR, Reynolds CF III, et al. Reducing suicidal ideation and depressive symptoms in depressed older primary care patients: a randomized controlled trial. JAMA. 2004;291(9):1081-1091.

Copyright 2017 American Medical Association. All rights reserved. 